\section{Benoît Coffin \\ Marc Lémann Raymond Jian}

\title{
Sensibilité viscérale digestive
}

Le tractus gastro-intestinal a une double innervation afférente : le système nerveux entérique qui permet une autonomie fonctionnelle partielle, en particulier les réflexes péristaltiques, et le système nerveux extrinsèque qui transmet l'information provenant de la muqueuse et de la musculeuse via les fibres afférentes vagales et spinales. Les récepteurs entériques et les fibres viscérales afférentes contiennent une grande variété de neuropeptides jouant peut-être un rôle spécifique dans la régulation des récepteurs et la transmission de l'information sensitive. Le dérèglement des récepteurs et de la transmission nerveuse ou des rétrocontrôles de l'information sensitive digestive pourraient être en cause dans les troubles digestifs fonctionnels (intestin irritable, dyspepsie fonctionnelle, colite ulcéreuse), que n'expliquent ni des anomalies des muscles lisses, ni des troubles psychologiques. L'efficacité sur la sensibilité digestive anormale d'analogues de la somatostatine et d'antagonistes des récepteurs de la sérotonine est en cours d'étude.

\section{ADRESSE}

B. Coffin : chef de clinique à la faculté de médecine de Paris, assistant des hópitaux. Hôpital Saint-Lazare et Inserm U.290, 107 bis, rue du Faubourg-Saint-Denis, 75010 Paris, France. M. Lémann : praticien hospitalo-universitaire à la faculté de médecine de Paris. R. Jian : professeur à la faculté de médecine de Paris, médecin des hôpitaux. Hôpital Saint-Louis, 1, avenue Claude-Vellefaux, 75475 Paris Cedex 10, France.

'innervation sensitive du tube digestif, particulièrement riche et complexe, reste encore mal connue. Son rôle est pourtant essentiel, compte tenu des multiples informations à traiter au cours des processus physiologiques digestifs et de la présence quasi constante de douleurs abdominales chez les malades consultant pour une affection gastrointestinale. Deux systèmes sensitifs (figure 1) coexistent et interagissent au niveau du tube digestif : (1) le système nerveux intrinsèque ou entérique qui autorise une certaine autonomie fonctionnelle au tube digestif; (2) les centres nerveux ganglionnaires médullaires et supramédullaires et les voies sensitives afférentes extrinsèques qui ont une fonction régulatrice sur le système nerveux intrinsèque et les viscères abdominaux. Une hiérarchie complexe permet le traitement des informations sensitives digestives selon différents niveaux d'intégration. Une très faible proportion de ces informations atteint le cortex, induisant des sensations beaucoup moins précises et spécifiques que les sensations somatiques. De nombreux travaux expérimentaux ont permis de mieux appréhender le fonctionnement et le rôle physiologique des afférences digestives. Chez l'homme, des difficultés méthodologiques ont longtemps limité les investigations, mais la mise en évidence de troubles de la sensibilité viscérale au cours des troubles fonctionnels intestinaux, a provoqué un regain d'inté- 


\section{RÉFÉRENCES}

1. Lémann M, Coffin B, Jian R. Sensibilité viscérale digestive : méthodes d'étude chez l'homme et implications physiopathologiques. Gastroenterol Clin Biol 1994: 18 (sous presse).

2. Wood JD. Neurophysiology of Auerbach's plexus and control of intestinal motility. Physiol Rev 1975 ; $55: 307-24$.

3. Andrews PLR. Vagal afferent innervation of the gastrointestinal tract. In : Cervero $F$, Morrisson JFB, eds. Progress in brain research, Vol. 67. New-york : Elsevier, 1986: 65-86.

4. Mei N, Condamin M, Boyer A. The composition of the vagus nerve in the cat. Cell Tissue Res $1980 ; 209$ : 423-31.

5. Grundy D, Scatcherd T. Sensory afferents from the gastrointestinal tract. In : Schultz SG, Wood JD, Rauner BB, eds. Handbook of physiology, Vol. 1, section 6. New York : Oxford University Press, 1989 : 593-620.

6. Mayer EA, Raybould HE. Role of visceral afferent mechanisms in functional bowel disorders. Gastroenterology $1990 ; 99: 1688$ 1704.

7. Kuo CD, Yang CGH, Yamaski DS, Krauthamer GM. A wide field electron microscopic analysis of the fibers constituents of the major splanchnic nerve in the cat. $J$ Comp Neurol 1982 ; 210 : 49-58.

8. Mei N. Recent studies on intestinal vagal afferent innervation. Functional implications. In : Kral JG, Powley TL, Brooks CM, eds. Vagal nerve function : behavioral and methodological considerations. New York : Elsevier, 1983: 199-206.

9. Melone J, Mei N. Intestinal effects of the products of lipid digestion on gastric electrical activity in the cat. Gastroenterology $1991 ; 100: 380-7$.

10. Notivol R, Mearin F, Azpiroz F, Malagelada JR. Epigastric symptoms are elicited by selective stimulation of " in series" and « in parallel " gastric mechanoreceptors. Gastroenterology 1990 ; 98 : A377.

11. Salt TE, Hill RG. Neurotransmitter candidates of somatosensory primary afferent fibers. Neuroscience $1983 ; 10: 1083-103$.

12. Moncada S, Higgs A. The L-Arginine nitric oxide pathway. $N$ Engl J Med 1993 ;

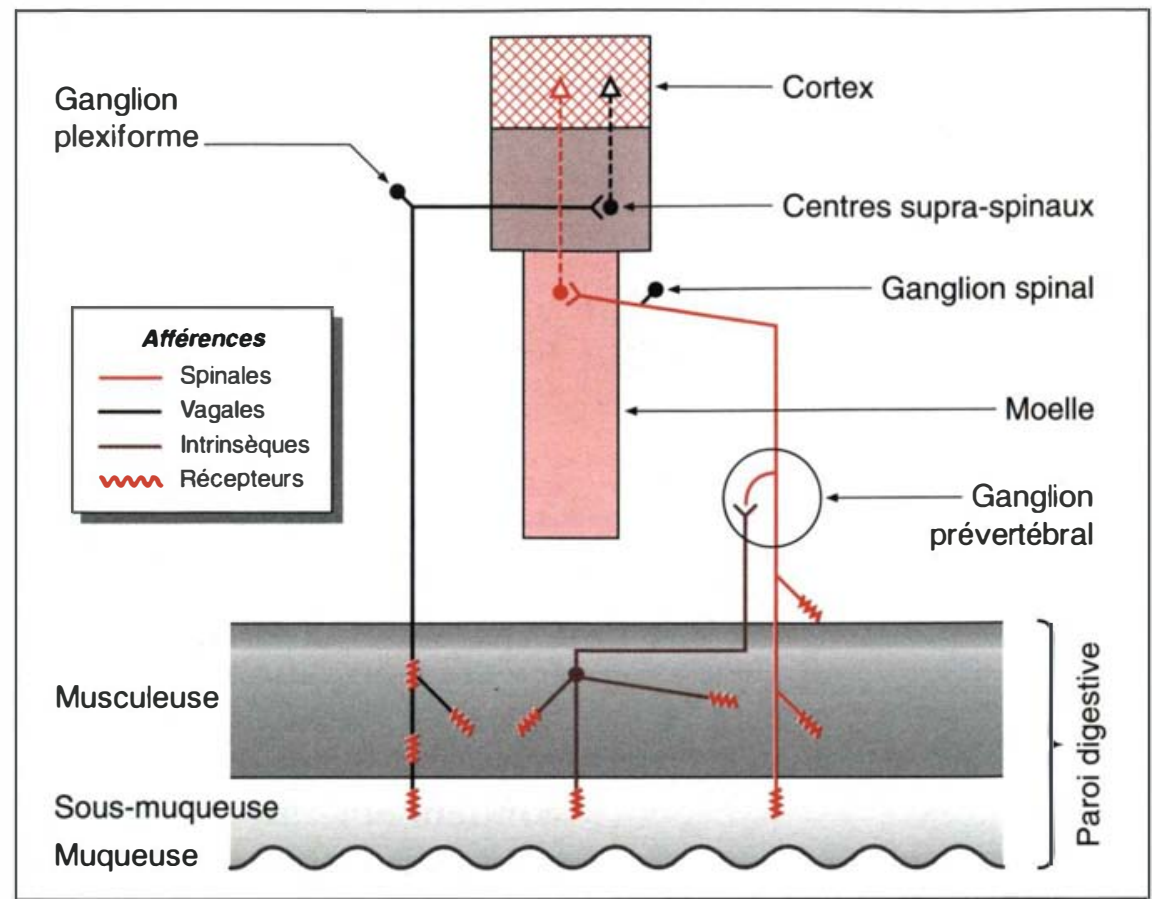

Figure 1. Représentation schématique des afférences viscérales digestives.

rêt pour ce domaine d'investigation. La sensibilité viscérale inconsciente est étudiée par la réponse effectrice (motrice le plus souvent) à des stimulations sensitives viscérales ou par le recueil de potentiels évoqués corticaux ou médullaires ; la sensibilité viscérale consciente est étudiée à l'aide d'échelles visuelles analogiques, ou de techniques de réflexologie [1].

\section{Données physiologiques}

\section{Le système nerveux intrinsèque}

Le système nerveux intrinsèque, situé dans la paroi du tube digestif, est composé de deux plexus intrapariétaux, le plexus myentérique et le plexus sous-muqueux. Présents de l'œesophage au canal anal, ces plexus sont organisés en structures laminaires, dans lesquelles des ganglions, contenant les corps cellulaires des neurones et des cellules gliales, sont reliés les uns aux autres par de très nombreux prolongements axonaux et dendritiques. Les synapses se présentent comme des renflements répartis tout le long des terminaisons axonales, assurant ainsi un nombre élevé de jonctions fonctionnelles sur un même axone. Les fibres nerveuses viennent directement au contact des cellules musculaires lisses et des autres structures des viscères digestifs (glandulaires, vasculaires, immunitaires). Parmi ce réseau de fibres nerveuses enchevêtrées, il est particulièrement difficile de différencier les afférences sensitives des efférences motrices [2].

\section{Le système nerveux extrinsèque}

Les afférences sensitives reliant le tube digestif aux centres nerveux supérieurs sont beaucoup mieux connues que celles du système nerveux intrinsèque. La plupart d'entre elles sont constituées de fibres non myélinisées de type $\mathrm{C}$ (diamètre < $1,2 \mu \mathrm{m})$. Elles se répartissent entre des afférences vagales qui cheminent dans le pneumogastrique et des afférences splanchniques ou spinales qui cheminent dans le système orthosympathique et dans le parasympathique pelvien (figure 1). Les affé- 
rences vagales assurent l'innervation sensitive de l'œsophage, de l'estomac, de l'intestin grêle et de la partie proximale du colon [3]. Leurs corps cellulaires sont situés dans le ganglion plexiforme, à proximité du bulbe. Leurs extrémités distales se situent au niveau du tronc cérébral, dans les noyaux du tractus solitaire, où elles s'articulent, soit avec des voies efférentes, soit avec un second neurone (deutoneurone) qui remonte vers les centres nerveux supérieurs. Les fibres afférentes représentent $80 \%$ du contingent neuronal du nerf vague [4]. Les afférences spinales cheminent dans les voies orthosympathiques assurant l'innervation sensitive de l'ensemble des viscères abdominaux, et dans les voies du système parasympathique pelvien assurant spécifiquement l'innervation de la partie distale du colon, du rectum et de l'anus [5]. Leurs corps cellulaires sont situés au niveau des ganglions spinaux. Leurs extrémités centrales sont localisées dans la corne postérieure de la moelle (laminae I et V) où elles s'articulent avec un deutoneurone qui transmet les informations sensitives vers les centres nerveux supérieurs [6]. Certaines fibres spinales s'articulent, au niveau des ganglions orthosympathiques prévertébraux, avec les neurones postganglionnaires des voies efférentes orthosympathiques. Le contingent sensitif des fibres spinales représente environ $20 \%$ des fibres orthosympathiques [7].

\section{Les récepteurs sensitifs}

En dehors des corpuscules de Paccini, situés au niveau du mésentère, aucune structure nerveuse spécifique n'a pu être attribuée aux récepteurs sensitifs digestifs qui se présentent donc sous la forme de terminaisons nerveuses libres. Des travaux électrophysiologiques chez l'animal et l'étude de boucles réflexes chez l'homme, ont toutefois permis d'individualiser, de façon assez précise, les fonctions réceptrices de ces terminaisons nerveuses sensitives. Les récepteurs intramuqueux, décrits au niveau de l'estomac, du duodénum et du colon [5], transmettent la majorité de leurs messages via le nerf vague. Il s'agit essentiellement des mécanorécepteurs, plus sensibles au frottement et à la déformation de la muqueuse digestive qu'à la distension. Certains d'entre eux répondent également à d'autres stimulations intraluminales (chimiques en particulier), et sont donc des récepteurs polymodaux. D'autres récepteurs muqueux sont hautement spécifiques [8,9], ne réagissant qu'à des stimulus précis (glucides, température, $\mathrm{HCl}$ ). Les mécanorécepteurs musculaires transmettent leurs messages via le nerf vague et les afférences spinales. Ils sont activés par la contraction musculaire (active) et par la distension intraluminale (passive). Lors d'une contraction isométrique (volume intraluminal constant) leur réponse est plus élevée que lors d'une

Tableau 1

\section{PRINCIPAUX MÉDIATEURS IMPLIQUÉS DANS LA SENSIBILITÉ VISCÉRALE ET AGENTS PHARMACOLOGIQUES CAPABLES DE DIMINUER LA VISCÉRO-PERCEPTION}

\begin{tabular}{|ll|}
\hline Médiateurs & Agents pharmacologiques \\
\hline Cholécystokinine (CCK) & Antagonistes de la CCK : loxiglumide \\
Somatostatine & Somatostatine : octréotide \\
Sérotonine (5HT) & Antagonistes $5 \mathrm{HT}_{3}$ : granisetron, \\
Opiacés : endorphines, enképhalines & odansetron \\
Tachykinines : substance P (SP) & Ligands opiacés kappa : fédotozine \\
Bombésine & Lidocaïne \\
Calcitonin gene-related peptide (CGRP) & Inhibiteurs calciques \\
Vasoactive intestinal peptide (VIP) & \\
Monoxyde d'azote (NO) & \\
& \\
\hline
\end{tabular}

$\mathrm{m} / \mathrm{s} \mathrm{n}^{\circ} 11$, vol. 10, novembre 94 contraction isotonique (pression maintenue constante par réduction du volume intraluminal). Lors d'une distension passive, leur seuil de réponse sera d'autant plus bas que le tonus musculaire du segment distendu est plus élevé [10]. Leur réponse se maintient pendant toute la durée de la stimulation (adaptation absente ou lente) et son seuil est bas (activation quasi permanente). Ces mécanorécepteurs se comportent donc comme des récepteurs "en-série" avec les cellules musculaires. Ils fournissent en permanence des renseignements sur l'activité musculaire du tube digestif qui, à son tour, influence leurs réponses [10]. Les mécanorécepteurs intraséreux transmettent la majorité de leurs messages via les afférences spinales. Leur seuil d'activation est élevé et leur réponse s'épuise rapidement lorsque leur stimulation est maintenue. Certains d'entre eux pourraient également être activés par des stimulations nociceptives chimiques ou thermiques.

\section{Neuropeptides des voies sensitives afférentes}

De nombreux peptides [11] sont synthétisés dans les afférences digestives, puis transportés et sécrétés au niveau des extrémités centrales et périphériques des neurones (Tableau I). Le monoxyde d'azote, récemment impliqué dans la neuromédiation efférente non-adrénergique-non-cholinergique, pourrait également participer à la neuromédiation sensitive digestive [12]. L'intégration centrale des messages afférents et le type des réponses efférentes qu'ils induisent dépendent de la nature des peptides libérés et de leur interaction au niveau des sites post-jonctionnels, mais il s'agit là de phénomènes encore très mal connus. Ces neuromédiateurs sont également libérés dans la paroi du tube digestif, soit par les dendrites des neurones intrinsèques, soit directement par les axones des fibres afférentes, via une "sécrétion antidromique". Les neuromédiateurs ainsi libérés participent à des fonctions effectrices (vasorégulation, sécrétion, contraction ou relaxation du muscle lisse digestif, inflammation...) et modulent à leur tour l'activité des 


\section{RÉFÉRENCES}

13. Frederickson RCA, Burgis V, Harrell CE, Edwarss JD. Dual actions of substance $\mathrm{P}$ on nociception : possible role of endogenous opioids. Science 1978 ; 216 : 1359-62.

14. Cramer WCM, Rademaker B. 5-HT3 receptors and visceral nociception : involvement and localization. Dig Dis Sci 1989 $34: 972$.

15. Hirst GDS, Holman ME, Mc Kirdy $\mathrm{HC}$ Two descending nerve pathways activated by distension of the guinea pig small intes tine. J Physiol (London) $1975 ; 244$ : 113-27.

16. Frantzides CT, Sarna SK, Matsumoto $T$, Lang IM, Condon RE. An intrinsic neural pathway for long intestino-intestinal inhibitory reflexes. Gastroenterology $1987 ; 92$ : 594-603.

17. Wiley J, Tatum D, Keinath R, Owyang $C$. Participation of gastric mechanoreceptors and intestinal chemoreceptors in the gastrocolic response. Gastroenterology 1988 ; gastrocolic

18. Azpiroz F, Malagelada JR. Vagally mediated gastric relaxation induced by intestinal nutrients in the dog. $A m J$ Physiol intestinal nutrients in

19. De Ponti, Azpiroz F, Malagelada JR Relaxatory response of canine proximal stomach to esophageal and duodenal dis tension. Importance of vagal pathways. Dig Dis Sci 1989; 34 : 873-81.

20. Kreulen DL, Szurszewski JH. Reflex pathways in the abdominal prevertebra ganglia: evidence for a colo-colonic inhibitory reflex. I Physiol (London) $1979 ; 295$ : 21-32.

21. Holzer $\mathrm{HH}$, Raybould HE. Vagal and splanchnic sensory pathways mediate inh bition of gastric motility induced by duode nal distension. Am J Physiol $1992 ; 262$. G603-8.

22. Iovino P, Azpiroz F, Dominguo E, Malagelada JR. The sympathetic nervous system gegulates sensory and reflex responses to gut stimuli in humans. Gastroenterology 1992; 102: A461.

23. Azpiroz F, Malagelada JR. Isobaric intestinal distension in humans : sensoria relay and reflex gastric relaxation. $A m J$ Physiol 1990 ; 258 : G202-7.

24. Cervero F. Somatic and visceral inputs to the thoracic spinal cord of the cat effects of noxious stimulation of the biliary récepteurs sensitifs. La complexité de ces phénomènes rend compte de certains effets parfois contradictoires de ces peptides sur la viscéro-perception. Ainsi, la substance $\mathrm{P}$ peut avoir, en fonction de la voie utilisée, une action analgésique ou hyperalgique [13], et la sérotonine se comporte comme un médiateur antinociceptif au niveau des centres médullaires et nociceptif au niveau périphérique [14].

\section{Boucles sensitivo-motrices}

Les informations recueillies et transmises par les voies sensitives intrinsèques et extrinsèques sont intégrées par des centres nerveux pour aboutir à des messages empruntant les voies nerveuses efférentes. Jusqu'à récemment, en particulier dans le domaine de la motricité, seules les voies efférentes ont été étudiées. En fait, des boucles sensitivo-motrices règlent de nombreuses fonctions digestives (motricité, sécrétions exocrines et endocrines, comportement alimentaire, vascularisation). Une commande efférente adaptée ne peut se concevoir sans un recueil et une transmission correcte d'informations provenant du tube digestif. Ces informations sont intégrées à différents niveaux : paroi intestinale, ganglions pré-vertébraux ou spinaux, centres supraspinaux. Peu d'entre elles parviennent au cortex, ou du moins, aboutissent à des sensations viscérales conscientes (viscéro-perception). Des travaux électro-physiologiques, effectués sur une anse intestinale isolée, ont permis d'explorer le rôle spécifique du système nerveux intrinsèque dans ces boucles de régulation. La stimulation de certaines afférences digestives par la distension déclenche des réactions motrices se succédant dans le temps : contraction musculaire en amont de la stimulation, relaxation musculaire en aval, puis contraction musculaire en aval [15]. Initialement décrit sur de très courtes distances, ce réflexe péristaltique peut, en fait, concerner des segments digestifs de plusieurs dizaines de centimètres [16]. Le système nerveux intrinsèque peut ainsi contrôler, de façon autonome, la progression du contenu endoluminal. Il en est probablement de même pour d'autres fonctions digestives (transport et vasomotricité locale) [6]. Les boucles de régulation empruntant le système nerveux extrinsèque aboutissent, au plan moteur, à des effets excitateurs ou inhibiteurs. La stimulation de chémorécepteurs et de mécanorécepteurs gastriques par l'alimentation, renforce l'activité motrice du côlon [17]. La stimulation de chémorécepteurs intestinaux [18] ou de mécanorécepteurs duodénaux [19] induit une relaxation gastrique. Les ganglions orthosympathiques prévertébraux se comportent comme un centre de contrôle, capable de régler certaines fonctions motrices, vasculaires ou glandulaires. Ils reçoivent, en effet, des synapses des neurones sensitifs spinaux qui, via la libération de substance $P$ et de CGRP (calcitonin gene-related peptide), peuvent activer des efférences orthosympathiques post-ganglionnaires [20].

Les fonctions spécifiques des voies sensitives vagales et spinales dans ces boucles de régulation ne sont pas encore clairement établies. Le nerf vague transmettrait les informations "physiologiques" alors que les voies spinales agiraient comme un signal d'alarme, que déclencheraient les stimulus nociceptifs [21]. En fait, ces deux voies sont inter-connectées et peuvent donc être impliquées dans les mêmes fonctions. Chez le rat, il a été démontré que l'inhibition de la motricité gastrique provoquée par la distension duodénale est relayée à la fois par le nerf vague et par les afférences splanchniques [21]. Chez l'homme, la stimulation élective du système orthosympathique abaisse le seuil d'apparition de la douleur provoquée par la distension, mais augmente aussi l'intensité de certains réflexes entéro-gastriques que l'on pensait uniquement médiés par le nerf vague [22].

\section{Viscéro-perception}

Comme nous l'avons souligné plus haut, peu de messages viscéraux parviennent jusqu'à la conscience et produisent alors des sensations vagues et non douloureuses, telles la faim, la satiété ou le besoin d'exonération fécale. Plus rarement, ces messages aboutissent à une sensation douloureuse correspondant à un 


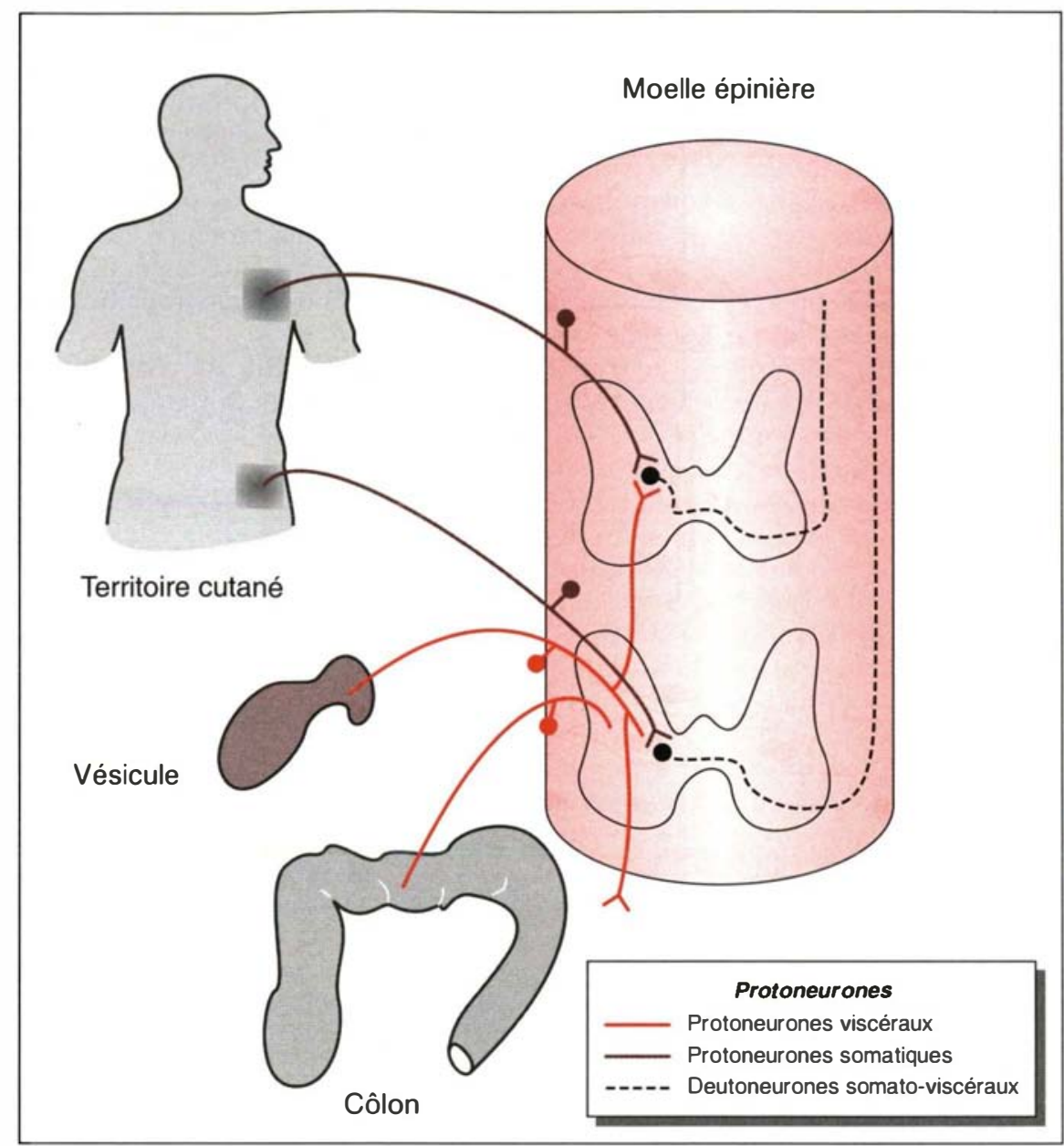

Figure 2. Phénomène de divergence-convergence. Dans cet exemple, les protoneurones innervant la vésicule biliaire, s'articulent avec plusieurs deutoneurones (expliquant le caractère "diffus" de la douleur viscérale), et convergent sur le même deutoneurone avec des protoneurones coliques et somatiques (expliquant le manque de spécificité et la projection cutanée de la douleur viscérale).

événement potentiellement nocif (nociception).

La nociception viscérale est beaucoup moins sélective que la nociception somatique. Certains stimulus, bien que potentiellement nocifs, tels une section ou une brûlure, n'entraînent pas de sensation douloureuse viscérale. Une distension duodénale progressivement croissante provoque d'abord une relaxation gastrique réflexe, puis une perception viscérale allant d'une sensation d'inconfort de plus en plus marquée à une douleur de plus en plus intense [23]. Ces dissociations suggèrent ment nocive, aboutirait à une sensation douloureuse, permettant une réponse adaptée de tout l'individu. Cette discrimination entre sensibilité viscérale consciente et inconsciente s'effectue probablement à un niveau spinal ou supra-spinal [25].

Les modalités de la viscéro-perception s'expliquent par l'anatomie fonctionnelle des voies nerveuses spinales, et en particulier par les phénomènes dits de convergence-divergence (figure 2). Les afférences sensitives viscérales ont une organisation rostro-caudale, mais des segments viscéraux distincts, voire de viscères digestifs différents, sont innervés par un même deutoneurone remontant vers les centres supérieurs. Cette convergence rend compte de la faible valeur localisatrice des sensations viscérales [25]. Par exemple, des douleurs pancréatiques, biliaires, coliques et gastriques peuvent avoir une expression clinique comparable (crampe épigastrique). De plus, les afférences sensitives viscérales s'articulent toujours avec des afférences somatiques sur les deutoneurones (neurones viscéro-somatiques). Cela explique que la plupart des douleurs viscérales soient rapportées à un territoire cutané. Enfin, un même neurone viscéral s'articule avec plusieurs deutoneurones, ce qui aboutit à une divergence des sensations viscérales sur trois à cinq métamères et explique la caractère diffus de la douleur viscérale.

La viscéro-perception est modulée par des voies inhibitrices et excitatrices. Certaines voies inhibitrices ont été caractérisées pour la sensibilité somatique. Il s'agit de contrôles inhibiteurs diffus prenant naissance au niveau supra-spinal [26] et mis en évidence, chez l'homme, par des phénomènes de réflexologie. La perception d'une stimulation électrique cutanée, et le réflexe spinal nociceptif de flexion (RIII) qu'elle déclenche, sont inhibés par une stimulation nociceptive somatique hétérotopique (phénomène de contre-irritation). Un phénomène identique est observé lorsque la stimulation nociceptive somatique est remplacée par une stimulation viscérale, ce qui suggère que les contrôles inhibiteurs diffus modulent également la viscéro-perception 


\section{RÉFÉRENCES}

25. Jaenig W. Spinal cord integration of vis ceral sensory systems and sympathetic nervous system reflexes. In : Cervero F, Morrisson JFB, eds. Progress in brain research, Vol. 67. New York : Elsevier, 1986 : 255-77.

26. De Broucker T, Cesaro P, Willer JC, Le Bars D. Diffuse noxious inhibitory controls in man. Involvement of the spinoreticular tract. Brain $1990 ; 113: 1223-34$.

27. Bouhassira D, Chollet R, Coffin B Lémann $M$, Le Bars $D$, Willer JC, Jian $R$. Inhibition of a somatic nociceptive reflex by gastric distension in man. Gastroenterolo gy 1994 (sous presse).

28. Coffin B, Azpiroz F, Malagelada JR Somatosensory modulation of gastrointestinal sensitivity in humans. Gastroenterology 1994 (sous presse)

29. Collet L, Meunier P, Duclaux R, CheryCroze $S$. Cerebral evoked potentials after endorectal mechanical stimulation in humans. Am J Physiol 1988 ; 254 : G477-82.

30. Ritchie J. Pain from distension of the pelvic colon by inflating a balloon in the irritable bowel syndrome. Gut 1973 ; 14 : 125-32.

31. Richter JE, Barish CF, Castell DO. Abnormal sensory perception in patients with esophageal chest pain. Gastroenterology $1986 ; 91: 845-52$.

32. Lémann $M$, Dederding JP, Flourié $B$, Franchisseur C, Rambaud JC, Jian R. Abnormal perception of visceral pain in response to gastric distension in chronic idiopathic dyspepsia. The irritable stomach syndrome. Dig Dis Sci 1991 ; 36 : 1249-54.

33. Prior A, Maxton DG, Whorwell PJ. Anorectal manometry in irritable bowel syndrome : difference between diarrhea and constipation predominant subjects. Gut $1990 ; 31: 458-62$.

34. Katz PO, Dalton CB, Richter JE, Wu WC, Castell DO. Esophageal testing in patients with non-cardiac chest pain or dysphagia : results of three years experience with 1161 patients. Ann Intern Med 1987 ; $106: 593-7$.

35. Coremans G, Tack I, Vantrappen G, Janssens J, Annese V. Is the irritable bowel really irritable ? Ital $j$ Gastroenterol $1991 ; 23$ (suppl 1) : 39-40.

36. Rao SSC, Davison PA, Bannister J, Holdsworth CD, Read NW. Anorectal sensitivity and reactivity in patients with ulcerative colitis. Gastroenterology $1987 ; 93: 1270$
[27]. A l'opposé, la stimulation de récepteurs cutanés peut entraîner une diminution de la perception viscérale douloureuse chez l'homme [28]. Des voies inhibitrices périphériques ou médullaires, voisines de celles décrites pour la sensibilité somatique (spinal gate control) et impliquant des opiacés endogènes, sont probablement à l'origine de ces rétrocontrôles négatifs de la transmission douloureuse viscérale. D'autres mécanismes sont, au contraire, susceptibles de renforcer la viscéro-perception. Ainsi, l'ischémie et l'inflammation abaissent le seuil de réponse des mécanorécepteurs digestifs [6]. Au-delà des contrôles périphériques et sous-corticaux, le cerveau est également impliqué dans la modulation de la viscéro-perception. Des facteurs émotionnels, cognitifs et un conditionnement peuvent, bien entendu, renforcer ou atténuer le niveau de perception d'une douleur viscérale. L'étude des potentiels évoqués cérébraux montre que certains messages, déclenchés par la stimulation d'afférences viscérales, parviennent au cerveau sans être perçus de façon consciente [29]. Des systèmes inhibiteurs très complexes allant du cerveau à la moelle sont impliqués dans ces phénomènes de facilitation ou d'inhibition de la viscéro-perception.

\section{Sensibilité viscérale et pathologie digestive}

\section{Description des anomalies sensitives}

Les troubles fonctionnels digestifs (TFD), qui par définition ne comportent aucune lésion morphologique ou histologique décelable, associent à des degrés divers des troubles du transit et des douleurs abdominales. Les anomalies objectives, les mieux individualisées au cours des TFD, concernent la motricité digestive. Cependant, elles ne sont présentes que chez environ $50 \%$ des patients. Des enregistrements prolongés montrent que leur survenue est souvent dissociée de celle des symptômes, et leur correction n'entraîne pas toujours d'amélioration clinique. Des stimulations physiologiques, échappant normalement à la viscéro-perception (contractions intestinales spontanées ou provoquées, reflux gastro-œsophagien physiologique, production et transit des gaz intestinaux), peuvent être à l'origine d'un inconfort ou de douleurs chez les malades atteints de TFD. Cela suggère, qu'à côté des troubles moteurs, des anomalies de la sensibilité viscérale sont impliquées dans la physiopathologie des TFD.

C'est à Ritchie [30] que l'on doit la première mise en évidence directe d'une anomalie de la viscéro-perception au cours du syndrome de l'intestin irritable (TFD associant des douleurs abdominales, des flatulences et des troubles du transit intestinal). Cet auteur a montré qu'une distension sigmoïdienne, provoquée par un ballonnet contenant $60 \mathrm{ml}$ d'air, induit une douleur abdominale chez $55 \%$ des malades explorés. Le même niveau de distension n'entraîne une douleur que chez $6 \%$ des sujets témoins. En revanche, la tension exercée sur le ballonnet par la paroi intestinale est identique chez les malades et les témoins, ce qui indique qu'un défaut de distensibilité (compliance) de l'intestin n'est pas en cause. Plusieurs travaux ont confirmé ces résultats et ont montré que l'anomalie sensitive affecte également le rectum et l'intestin grêle. Plus récemment, Richter et al. [31] ont mis en évidence un abaissement du seuil douloureux à la distension dans les douleurs thoraciques pseudo-angineuses. La distension d'un ballonnet œsophagien par $8 \mathrm{ml}$ d'air (qui n'est pas perçue par les sujets témoins) reproduit chez $50 \%$ des malades leur douleur habituelle. Ici encore, un défaut de compliance ne peut expliquer cette anomalie. Ce trouble sensitif semble jouer un rôle plus important que les classiques troubles moteurs œsophagiens (spasmes diffus et œsophage cassenoisettes) dans la physiopathologie de ces douleurs pseudo-angineuses. Nous avons récemment mis en évidence un abaissement du seuil douloureux à la distension gastrique dans la dyspepsie chronique idiopathique, TFD associant à des degrés divers satiété précoce, tension épigastrique postprandiale, sensation de digestion lente (figure 3). Une stase gastrique et une hypersensibilité viscérale à la distension sont présentes 


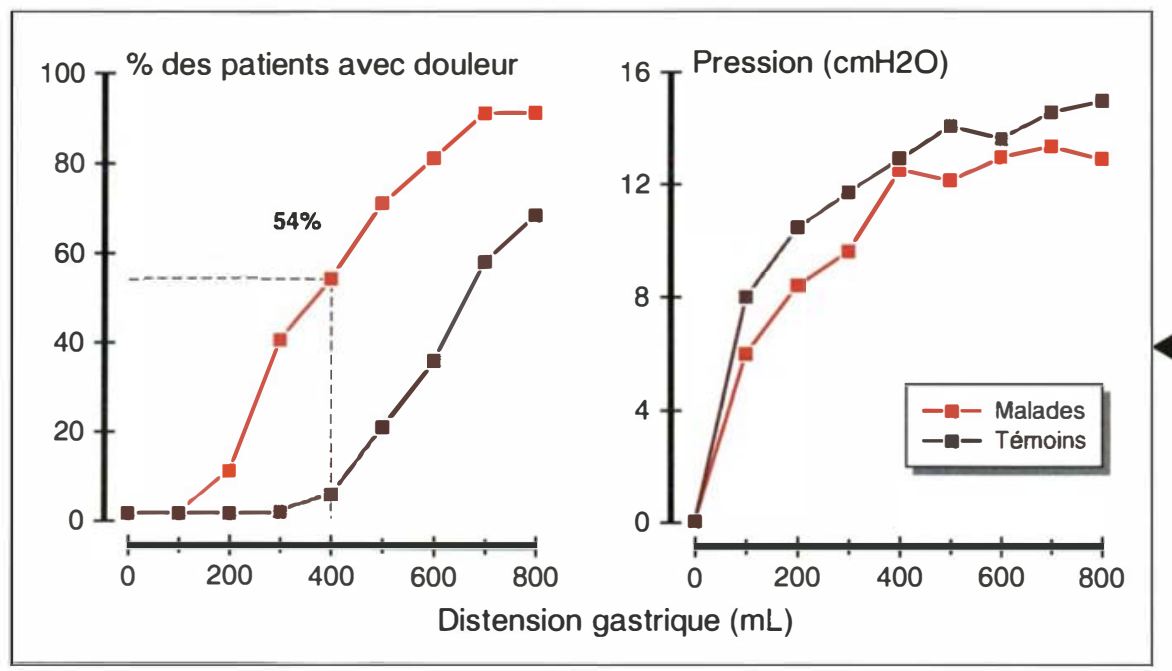

Figure 3. Résultats du test de distension gastrique chez des sujets témoins et des patients souffrant de dyspepsie chronique idiopathique. La courbe de douleur-distension est significativement déplacée vers la gauche chez les dyspeptiques (hypersensibilité viscérale); la courbe pression-volume n'est pas modifiée (compliance normale). isolément ou, plus souvent associées, chez près de $90 \%$ des patients dyspeptiques [32]. Des anomalies de la viscéro-perception sont également présentes au cours des troubles du transit intestinal. Des tests de distension ont objectivé une diminution de la sensibilité rectale dans certaines constipations idiopathiques et dans certains cas d'incontinence fécale. A l'opposé, une hypersensibilité rectale a été mise en évidence dans certaines formes de diarrhées idiopathiques [33].

Ces résultats, remarquablement homogènes, montrent : (1) qu'il existe au cours de tous les types de TFD un abaissement du seuil douloureux à la distension; (2) que la fréquence de cette anomalie est d'environ $50 \%$ pour des niveaux de distension n'induisant pas de douleur chez des sujets témoins ; (3) qu'il s'agit d'un véritable trouble sensitif et non d'anomalies secondaires à un trouble moteur ou un défaut de compliance. L'anomalie du seuil de perception viscérale concerne, soit spécifiquement la sensation douloureuse, soit l'ensemble des sensations provoquées par la distension. Les anomalies sensitives ne concernent probablement pas que la distension viscérale. Ainsi, une hypersensibilité à l'acide, à l'origine du test de Bernstein, peut être présente au cours du reflux gastro-œso- phagien (œsophage acido-sensible) ou des douleurs thoraciques pseudoangineuses [34] et une hypersensibilité aux acides biliaires et aux acides gras a été rapportée dans le syndrome de l'intestin irritable [35].

L'existence et le rôle physiopathologique d'anomalies de la sensibilité viscérale digestive a été peu étudiée en dehors des TFD. Un abaissement du seuil douloureux à la distension rectale a été mis en évidence dans la recto-colite hémorragique [36]. Certains troubles du comportement alimentaire pourraient également comporter des anomalies de la viscéro-perception aboutissant à des perturbations des sensations de faim et de satiété [37]. Enfin, chez l'animal, la destruction de certaines afférences digestives potentialise les effets gastro-toxiques de l'alcool [38], ce qui suggère une implication des troubles sensitifs viscéraux dans le phénomène de cytoprotection.

\section{Origine des anomalies sensitives}

Les anomalies psychologiques ou psychiatriques, mises en évidence dans les TFD, pourraient être à l'origine des troubles de la viscéro-perception. Par exemple une tendance névrotique à qualifier de douloureuses des sensations normales, ou une tendance hypocondriaque à médicaliser des sensations physiolo- giques, pourraient expliquer ces anomalies. Cette hypothèse est étayée par le polymorphisme des symptomes fonctionnels qui débordent largement la sphère digestive, par leur bénignité (absence de retentissement sur l'état général et de complications évolutives), et par le fait qu'une minorité des sujets se plaignant de TFD, très fréquents dans la population générale, consulte. En fait, il n'existe pas de corrélation entre la présence et l'intensité des anomalies psychologiques (mesurées par des tests psychométriques) et celles des anomalies sensitives. Dans notre étude sur la dyspepsie chronique, les anomalies psychométriques étaient même significativement moins intenses dans le sous-groupe des patients ayant une anomalie de la viscéro-perception [32]. L'excellente reproductibilité de l'anomalie du seuil douloureux, l'absence de symptômes provoqués par une dilatation fictive, l'absence d'hyperesthésie somatique ou viscérale en dehors du territoire concerné par le TFD [39] et la persistance d'une discrimination normale entre stimulations nociceptives qualitativement différentes [40] plaident également contre l'origine psychogène de ces troubles sensitifs.

Il est donc vraisemblable que ces anomalies correspondent, au moins en partie, à une véritable atteinte 


\section{RÉFÉRENCES}

37. Geliebter A, Melton PM, Mc Cray RS Gallagher DR, Gage D, Hashim SA. Gastric capacity, gastric emptying, and test-meal intake in normal and bulimic women. $A m J$ Clin Nutr 1992 ; $56: 656-61$.

38. Holzer P, Lippe TH. Stimulation of afferent nerve endings by intragastric capsaicin protects against ethanol-induced damage of gastric mucosa. Neuroscience $1988 ; 27: 981-7$.

39. Mearin F, Cucala M, Apiroz F, Malagelada JR. The origin of symptoms on the brain-gut axis in functional dyspepsia. Gastroenterology 1991 ; 101 : 999-1006.

40. Accarino A, Azpiroz F, Malagelada JR. Receptor versus suprareceptor level of gut sensory disfunction in the irritable bowel syndrome. Gastroenterology $1992 ; 102$ : A413.

41. Plourde V., Lembo T, Shui Z, Parker J, Mertz H, Taché Y, Sytnik B, Mayer E. Effects of the somatostatin analogue octreotide on rectal afferent nerves in humans. Am J Physiol 1993 ; 265 : G742-51.

42. Moriarty KJ, Dawson AM. Functional abdominal pain : further evidence that whole gut is affected. $B r$ Med $J 1982$; 284 : 1670-2.

43. Hasler WL, Soudah HC, Owyang C. A somatostatin analogue inhibits afferent pathways mediating perception of rectal distension. Gastroenterology 1993; 104 : $1390-7$.

44. Prior A, Read NW. Reduction of rectal sensitivity and post-prandial motility by granisetron, a $5 \mathrm{HT}_{3}$-receptor antagonist, in patients with irritable bowel syndrome. Aliment Pharmacol Ther 1993 ; 7 : 175-80.

45. Hammer J, Phillips SF, Talley NJ, Camilleri M. Effect of a $5 \mathrm{HT}_{3}$-antagonist (ondansetron) on rectal sensitivity and compliance in health and the irritable bowel syndrome. Aliment Pharmacol Ther $1993 ; 7: 543-51$

46. Coffin B, Azpiroz F, Malagelada JR. Mécanismes des réponses sensorielles à des stimulations viscérales et somatiques. Gastroenterol Cin Biol 1991; 15:28A.

47. Coffin B, Jian R, Lémann M, Fraitag B, Van Egroo LD, Franchisseur C, Rambaud JC. Fedotozine increases threshold of discomfort to gastric distension in healthy subjects. Gastroenterology $1992 ; 102$ : neurologique, affectant un ou plusieurs des maillons de la chaîne complexe transmettant et intégrant les messages sensitifs provenant du tube digestif (figure 1). Des travaux récents suggèrent que certains mécanorécepteurs musculaires sont directement impliqués dans les troubles sensitifs digestifs [41]. Moriarty et al. [42] ont montré que la projection cutanée d'une douleur, provoquée par une distension colique, est plus étendue chez les patients ayant un syndrome de l'intestin irritable que chez des sujets témoins. De même, le segment digestif dont la stimulation induit une douleur projetée sur un des quadrants abdominaux, est anormalement étendu chez ces malades. Ces constatations suggèrent l'existence d'anomalies des rétrocontrôles qui limitent, au niveau des voies nerveuses extrinsèques et des centres spinaux et supraspinaux, les phénomènes de divergence/convergence de la douleur.

\section{Perspectives thérapeutiques}

La découverte d'anomalies sensitives directement impliquées dans la physiopathologie des TFD offre d'intéressantes perspectives thérapeutiques. Plusieurs des médiateurs de la sensibilité viscérale décrits plus haut sont en cours d'étude (Tableau 1). Trois classes thérapeutiques ont fait, à ce jour, l'objet d'études chez l'homme. Les travaux les plus prometteurs concernent la somatostatine, et plus particulièrement son analogue à effet retard, l'octréotide. L'octréotide élève le seuil douloureux de la distension rectale chez des sujets témoins et chez des patients atteints d'un syndrome de l'intestin irritable [41, 43]. A la dose utilisée, cet analogue n'a pas d'effet sur la sensibilité cutanée et sur la compliance rectale. Des travaux récents [41] suggèrent que la somatostatine agit sur les récepteurs muqueux et les voies afférentes correspondantes sans mettre en jeu les opiacés endogènes. Les antagonistes des récepteurs de la sérotonine, de type $5 \mathrm{HT}_{3}$, ont donné des résultats discordants au niveau de la sensibilité rectale chez des patients atteints d'un syndrome de l'intestin irritable $[44,45]$ et semblent inefficaces au niveau de l'estomac [46]. La fédotozine, agoniste périphérique des récepteurs opiacés kappa, élève le seuil douloureux lors de distensions gastriques chez des sujets témoins, mais ce résultat doit être confirmé chez des patients ayant une anomalie de la viscéro-perception [47]. Aucune étude clinique contrôlée testant de manière objective les effets de l'administration prolongée de ces substances sur les anomalies sensitives digestives et les symptômes correspondants n'est actuellement disponible.

\section{Conclusion}

L'étude de la sensibilité viscérale digestive a été négligée jusqu'à ces dernières années alors que beaucoup des symptômes rencontrés en gastro-entérologie évoquent des anomalies dans ce domaine. Les résultats obtenus dans les TFD montrent que la viscéro-sensibilité est une hypothèse physiopathologique au moins aussi intéressante que celle concernant les voies nerveuses efférentes et le muscle lisse digestif. Audelà de la viscéro-perception et de ses troubles, des anomalies des afférences nerveuses viscérales pourraient être impliquées dans le dysfonctionnement de nombreuses boucles sensitivo-efférentes inconscientes. Il est ainsi possible que certaines anomalies motrices ou sécrétoires digestives soient secondaires à des perturbations des systèmes de recueil des informations. La signification clinique des anomalies de la sensibilité viscérale consciente ou inconsciente, et l'intérêt pratique de leur mise en évidence, ne pourront être établis que par des études pharmacologiques et cliniques testant, chez l'homme, des substances capables d'agir spécifiquement sur ces troubles

\section{TIRÉS A PART}

B. Coffin. 


\section{Summary}

Gastrointestinal tract sensibility

This review analyzes current concepts of visceral afferent pathways and their role in the perception, modulation and integration of stimuli arising from the gastrointestinal (GI) tract. It also analyzes the clinical evidence supporting that altered visceral afferent mechanisms have a role in the pathogenesis of functional bowel disorders.

The GI tract has a dual afferent innervation: the enteric nervous system, which is a complex network of ganglia and nervous fibers and allows partial functional autonomy, especially in the regulation of peristaltic reflexes, and the extrinsic nervous system which transmits sensiti- ve information from the mucosa and muscular wall to the medullar and supramedullar centers of control, via the vagal and spinal afferent fibers. Information from the gut is collected by specific or polymodal receptors. Visceral afferent fibers contain a wide variety of neuropeptides which might have a specific function in the regulation of receptors or the transmission of sensory information. Exploration of visceral afferent fibers is difficult in humans, and involves the study of reflex loops, especially sensorymotor reflexes. Both vagal and spinal afferents can transmit physiological and noxious information to the upper centers of control, but only a few of them are conscious.

In human pathology, the hypersen- sitivity of the GI tract to various stimuli has been clearly demonstrated in different functional bowel disorders, such as the irritable bowel syndrome, non cardiac chest pain and functional dyspepsia, and in ulcerative colitis. This hypersensitivity is not due to alterations in the mechanical properties of the smooth muscle. Neither does it seein to be related to psychologic abnormalities. Up-regulation of gut receptors and perturbation of the neural transmission or retro-control of sensitive information by afferent fibers, might be involved in this GI tract hypersensitivity. Drugs able to reduce visceral sensitivity are required and, in this connection, somatostatin and $5 \mathrm{HT}_{3}$ antagonists are currently being evaluated in man. 